\title{
Clinical significance of preoperative liver stiffness measurements in primary HBV-positive hepatocellular carcinoma
}

\author{
Min Qi ${ }^{1}$, Yu Chen², Guo-Qiang Zhang ${ }^{3}$, Yu-Juan Meng ${ }^{4}$, Fu-Li Zhao'1, Jing Wang1 \& Jun \\ $\mathrm{Ma}^{*}, 5$ \\ ${ }^{1}$ Department of General Medical, Luoyang Central Hospital Affiliated to Zhengzhou University, No. 288, Zhongzhou Middle Road, \\ Luoyang 471009, PR China \\ ${ }^{2}$ Liver Intensive Care Unit, Beijing You'an Hospital Affiliated to Capital Medical University, No. 8, Xi Tou Tiao, Youanmen Wai, \\ Fengtai District, Beijing 100069, PR China \\ ${ }^{3}$ Department of Infectious Disease, Luoyang Central Hospital Affiliated to Zhengzhou University, No. 288, Zhongzhou Middle \\ Road, Luoyang 471009, PR China \\ ${ }^{4}$ Clinical Laboratory Center, Luoyang Central Hospital Affiliated to Zhengzhou University, No. 288, Zhongzhou Middle Road, \\ Luoyang 471009, PR China \\ ${ }^{5}$ Institute of Digestive Diseases, The Second Affiliated Hospital of Zhengzhou University, No. 2, Jingba Road, Zhengzhou 450014, \\ PR China \\ * Author for correspondence: Tel.: +0086 371 450014; Fax: +0086 371 450014; majun885@sina.com
}

\begin{abstract}
Aim: To analyze clinical significance of preoperative liver stiffness measurement (LSM) by FibroScan in postcurative resection hepatitis B virus (HBV) related hepatocellular carcinoma (HCC). Patients \& methods: A total of 263 patients underwent preoperative LSM and curative operation for primary HBV-positive HCC were enrolled. The correlation between preoperative LSM and survival was analyzed. Results: All patients were stratified into two groups using the optimal cut-off value (13.2 kPa) of LSM using the receiveroperating characteristic. Patients with an LSM $\geq 13.2 \mathrm{kPa}$ had poorer overall survival (median, 61.3 vs 48.2 months, hazard ratio: $0.15 ; \mathrm{p}=0.009$ ) and recurrence-free survival (median, 60.4 vs 47.0 months; hazard ratio: $0.32 ; p=0.011$ ) than patients with an LSM $<13.2 \mathrm{kPa}$ and LSM also have been confirmed as independent predictor for survival for HCC. Discussion: This could potentially guide patient stratification and individualized treatment. Conclusion: Preoperative LSM can be considered as an independent prognostic factor for HBV-positive HCC after curative resection.
\end{abstract}

First draft submitted: 21 June 2017; Accepted for publication: 21 August 2017; Published online: 30 November 2017

Keywords: HBV • hepatocellular carcinoma • liver stiffness measurement • prognosis • survival

Hepatocellular carcinoma (HCC) is the most common malignancy in China, its morbidity and mortality remain high owing to the increasing amount of hepatitis B virus (HBV) carriers [1-3]. Although improvement of comprehensive treatment and advances in surgical techniques have benefited HCC patients, the long-term prognosis of HCC remains unsatisfactory mainly due to recurrence and relapse after surgical resection, especially for HBVpositive HCC patients [4,5]. Liver fibrosis or cirrhosis resulted from chronic HBV infection and inactive HBV carrier status has shown a close relationship with postoperative mortality, complications and survival of HCC [6-8]. Furthermore, it was known that HBV status was associated with outcome of HBV patients, such as HBV viral load, HBV-DNA. However, in China, not all patients have had an HBV-DNA evaluation, so HBsAg positive was always used as the marker of HBV status. Thus, preoperative assessment of accurate quantification of the degree of fibrosis is essential for predicating prognosis and guiding surveillance. However, currently, liver fibrosis is mainly evaluated though liver biopsy or postoperative historical examination, which shows invasive characteristics, potential for complications and diagnostic differences among pathologists $[9,10]$. Hence, novel and non-invasive methods of pretreatment fibrosis assessment that are highly reproducible and technically feasible and pathologist independent are required.

Future Medicine 
Recently, liver stiffness measurement (LSM) using transient elastography (FibroScan) has been known as a novel, non-invasive tool for evaluating the degree of liver fibrosis with high reproducibility and reliability [11-13]. It has been revealed that LSM is useful to predict the development of HCC in patients with chronic liver disease [14,15]. Furthermore, LSM can also predict postoperative complication and recurrence for patients that underwent surgery for HCC [16,17]. However, the role of preoperative LSM in prediction of postoperative survival and clinical staging has not been elucidated. Hence, in this study, we try to assess significance of preoperative LSM in prognosis predicting and clinical staging for HBV-positive HCC patients that underwent curative resection.

\section{Materials \& methods}

\section{Patients}

Two hundred and sixty three patients with primary HCC undergone curative resection at the Second Affiliated Hospital of Zhengzhou University were enrolled in this study between 1 January 2010 and 1 January 2015. Inclusion criteria included histologically confirmed and curative resection, in other words, 'operable' HCC with HBsAg positive, more than 18 years of age and life expectancy of more than 6 months. Patients who had acute and severe preoperative infection, malignant hematologic conditions or adjuvant treatments were excluded from this study. Patients for whom clinicopathologic data and LSM measurement were unavailable and unreliable were also excluded. Patients that had died from causes unrelated to HCC during the follow-up were also excluded. Clinicopathological characteristics of all patients were collected from the medical records by one surgeon and checked by another surgeon, including gender, age, tumor size, liver cirrhosis, Child-Pugh score, hepatitis B surface antigen (HBsAg), AFP level, Barcelona Clinic Liver Cancer (BCLC) stage, TNM stage, tumor site and size, portal vein invasion (PVTT), pathological differentiation and operation type. Histopathological and TNM staging were evaluated though postoperative histopathological examination and clinical assessment, respectively. Liver cirrhosis was diagnosed by postoperative histological evaluation. Hepatic resections were described using Brisbane 2000 terminology [18]. Major resections were defined as hemihepatectomy and resection of more than three liver segments, while minor resection included localized resection and single-segment resection. For patients with Cheng's classification for PVTT type I and II, thrombectomy was performed, and the PVTT was resected en bloc with the specimen. Tumor recurrence was limited to intrahepatic relapse. Otherwise, we followed the methods of Li et al. [19].

\section{Liver stiffness measurement}

LSM by transient elastography was performed using a FibroScan502 (Echosens, Paris, France) with the M-probe and L-probe. Patients were placed in a supine position with the right hand with the right arm in maximum abduction for right intercostal scanning. LSM was only performed by one experienced technician, who was blind to the patients' information. The results are expressed as kilopascals $(\mathrm{kPa})$. According to the instructions provided by the manufacturer, when at least ten effective measurements were obtained with effective measurement at $60 \%$ or higher and interquartile range at less than $30 \%$, such measurements were defined as reliable. The median value of successful measurements was considered as a representative of the LSM value for a given patient.

\section{Follow-up}

After discharge, all patients were followed up in regular style through outpatient visit including physical examination and laboratory examinations including AFP every 3 months for the first 2 years, every 6 months for the next 3 years and once annually thereafter. Enhanced abdominal CT or MRI scans were performed generally every 12 months. Clinical follow-up lasted from the date of surgery to either the time of death or June 2016. The death was defined as disease-specific death. This study was approved by the Ethics Committee of the Second Affiliated Hospital of Zhengzhou University. Written informed consent was obtained from individual patients.

\section{Statistical analysis}

Statistical analyses were performed by using SPSS 20.0 (IBM, NY, USA). p $<0.05$ (two-sided) was considered as statistically significant. The optimal cut-off values for the LSM were determined through receiver-operating characteristic (ROC) curve analysis. Overall survival (OS) was accurately defined as the duration from date of surgery to death while recurrence-free survival (RFS) was calculated by the time of surgery to tumor recurrence. The $\chi^{2}$-square test or Fisher's exact test was used to analyze the association between qualitative variables and LSM while quantitative values were analyzed by independent Student's t-test. The OS and RFS and survival curve was 
Table 1. Correlation between the preoperative liver stiffness measurement and clinicopathological characteristics.

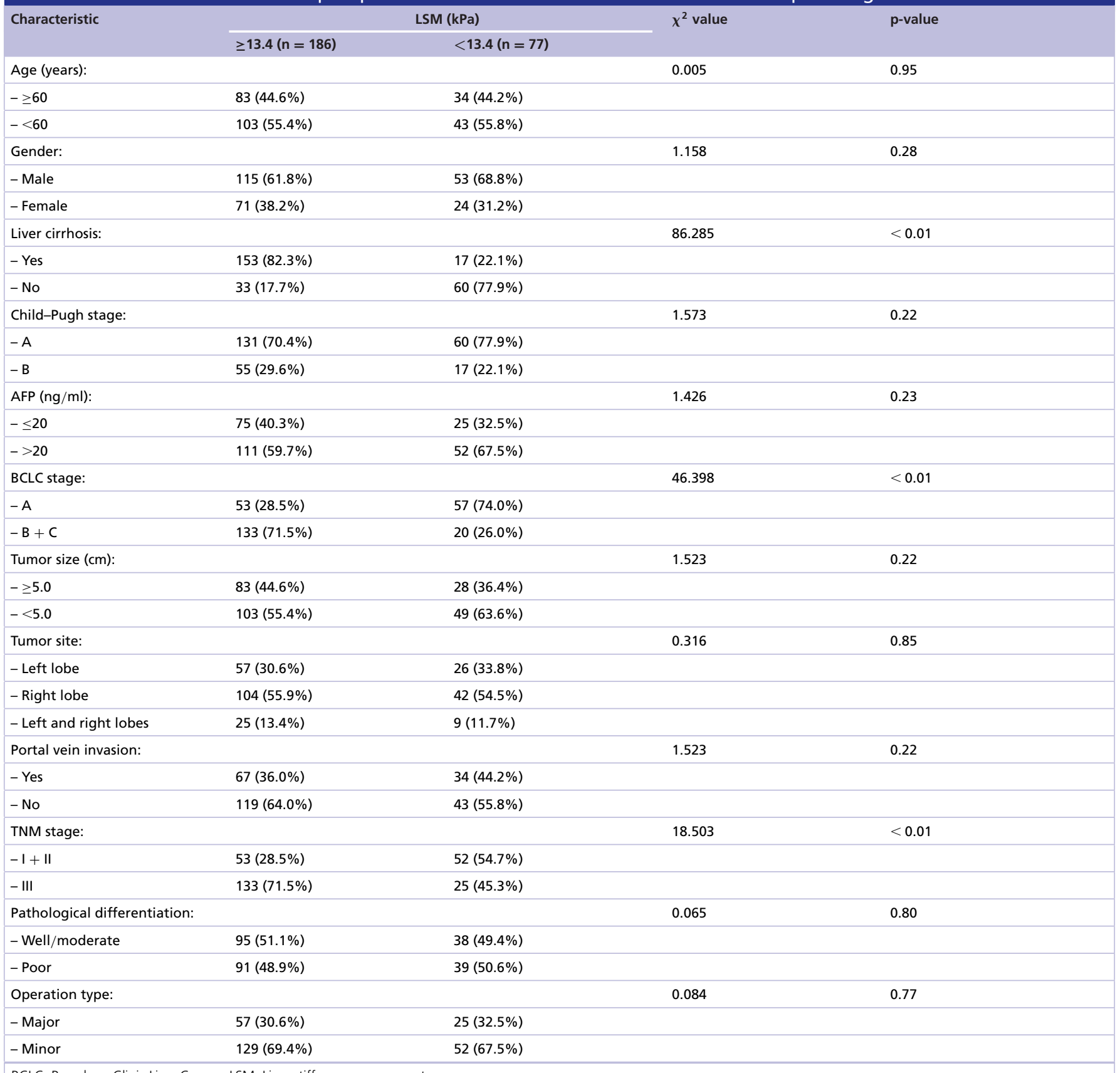

BCLC: Barcelona Clinic Liver Cancer; LSM: Liver stiffness measurement.

studied in Kaplan-Meier analyses by using the log-rank test. The Cox regression model was used to assess the hazard ratio (HR) and multivariate analysis.

\section{Results}

Baseline characteristics

The correlation between the preoperative LSM and clinicopathological characteristics is shown in Table 1. Total of 263 patients with primary HCC in this study include 168 (63.9\%) males and 95 (36.1\%) females, with $58 \pm 1.6$-year average age. According to the TNM staging methods, there were 105 (39.9\%) patients with stage I or II tumor, $158(60.1 \%)$ patients with stage III tumor. Otherwise, $110(41.8 \%)$ patients had BCLC stage A tumor and 153 (58.2\%) patients with BCLC stages B and C disease. For pathology, 133 (50.6\%) patients suffered 


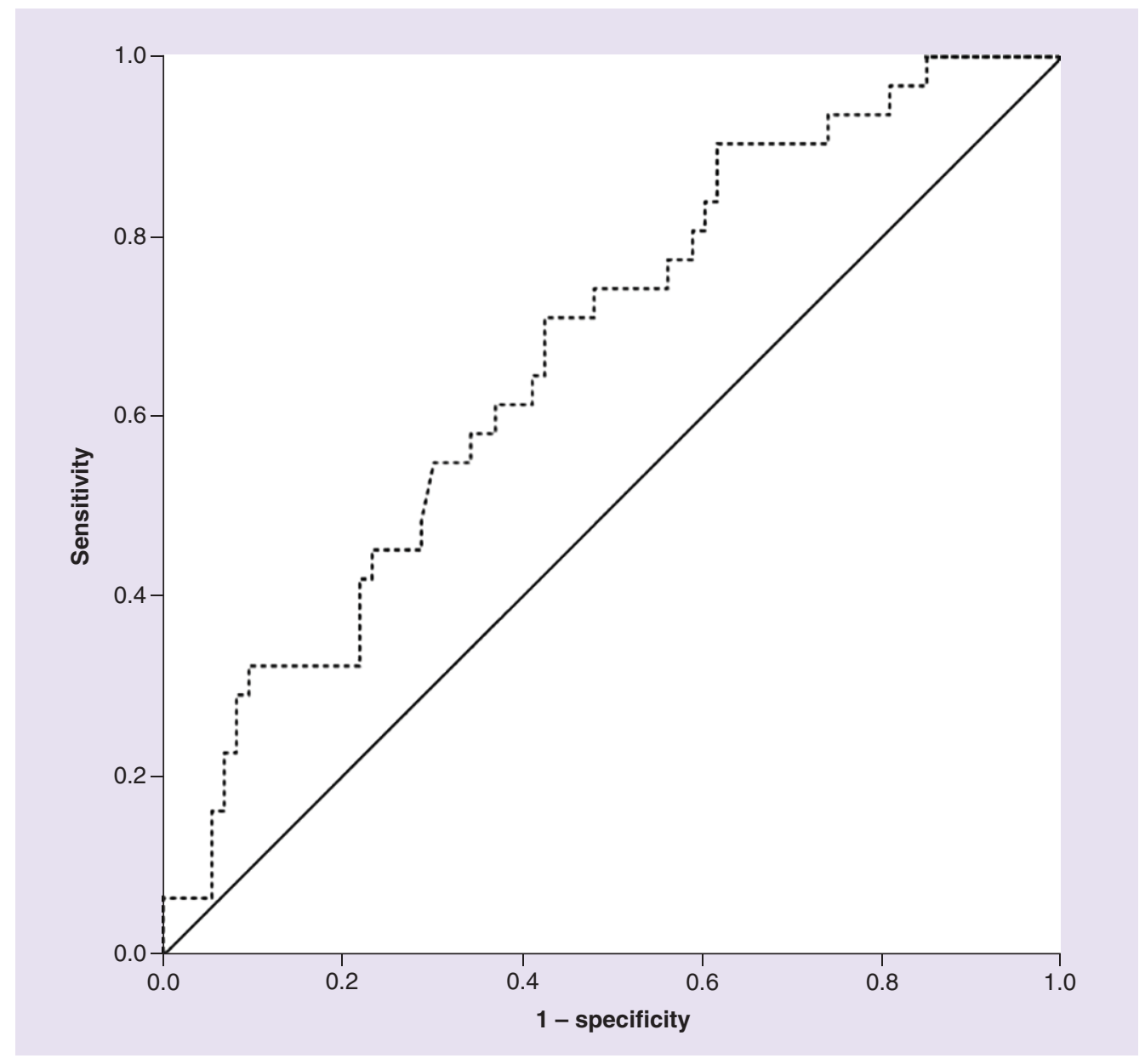

Figure 1. Receiver-operator characteristic curve for liver stiffness measurement.

from well/moderate differentiation of the tumor whereas 130 (49.4\%) patients suffered from poor differentiation. Median preoperative LSM was 13.4 (4.1-45.2) $\mathrm{kPa}$, which was positively significantly associated with liver cirrhosis, TNM stage and BCLC stage $(\mathrm{p}<0.05)$. However, there was no statistically significant association between LSM and other clinicopathological characteristics.

\section{Prognostic significance of LSM for HCC}

According to the ROC curve analysis, the optimal cut-off level for the LSM predicting OS of HCC patients was $13.2 \mathrm{kPa}$ using a sensitivity of $66.3 \%$ and a specificity of $84.7 \%$ as optimal conditions, respectively. The area under the curve was 0.672 with a $95 \% \mathrm{CI}$ for the area between 0.563 and 0.781 (Figure 1).

At the end point of this study, 83 (31.5\%) out of all 263 patients studied had died, including $18(23.4 \%)$ in 77 patients with a LSM $<13.2 \mathrm{kPa}$ and $65(34.9 \%)$ out of 186 patients with a $L S M \geq 13.2 \mathrm{kPa}$, respectively. Eighty-five (45.7\%) in group of 186 patients with a LSM $\geq 13.2 \mathrm{kPa}$ had tumor relapse while 27 (31.2\%) in patients group with LSM $<13.2 \mathrm{kPa}$ suffered from recurrence. Kaplan-Meier univariate survival analysis showed PVTT, TNM stage, BCLC stage, histological differentiation type and preoperative LSM were related to postoperative survival of HCC patients. Among these prognostic factors, LSM $\geq 13.2 \mathrm{kPa}$ was associated with a shorter OS of HCC patients. The mean OS of patients with preoperative LSM $<13.2 \mathrm{kPa}$ was 61.3 months, which was statistically significantly higher than 48.2 months of those with a LSM $\geq 13.2 \mathrm{kPa}(\mathrm{p}=0.009$; Table $2 \&$ Figure 2). Furthermore, we obtained a similar result in the multivariate analysis for OS (HR: 0.15; 95\% CI: 0.09-0.25; p = 0.022; Table 2). Otherwise, in a univariate analysis of RFS, patients with high LSM also had shorter mean RFS than patients with low LSM (60.4 vs 47.0 months; $\mathrm{p}=0.011$ ), and the low LSM was also confirmed as a significant independent predictor for RFS in multivariate analysis (HR: 0.32; 95\% CI: 0.04-1.02; $\mathrm{p}=0.032$; Table 3). 
Table 2. Univariate and multivariate analyses of the prognostic factors of overall survival for hepatocellular carcinoma.

Characteristics

Univariate

MS (months)

n

Age (years):

$-\geq 60$

$-<60$

Gender:

- Male

- Female

Liver cirrhosis:

-Yes 170

- No

Child-Pugh stage:

$-A$

$-B$

$\operatorname{AFP}(\mathrm{ng} / \mathrm{ml})$ :

$-\leq 20$

$->20$

BCLC stage:

- A 110

$-B+C$

Tumor size $(\mathrm{cm})$ :

$-\geq 5.0$

$-<5.0$

Tumor site:

- Left 83

- Right

- Left and right

Portal vein invasion:

- Yes

- No

Tumor stage:

$-1+$ II

- III

Pathological differentiation:

- Well/moderate

- Poor

Operation type:

- Major

- Minor

LSM (kPa):

$-\geq 13.2$

$-<13.2$

56.3

55.8

54.3

63.3

56.5

58.7

53.4

56.8

64.2

65.7

$163 \quad 65.7$

$10 \quad 65.4$

$153 \quad 52.6$

63.5

62.2

63.1

61.6

57.8

34

45.6

$162 \quad 65.4$

105

$158 \quad 49.2$

$\left(\frac{10}{2}+7\right.$

68.7

44.6

58.3

57.2

48.2

61.3

$\begin{array}{ll} & p-v a l u e \\ 0.102\end{array}$

0.486

0.524

$<0.001$

7.51

$2.87-10.54$

0.001

0.523

0.632

0.042

0.62

0.31-0.95

0.036

$95 \% \mathrm{Cl}$

p-value

por

0.531

0.273

0.031

3.80

$1.23-9.55$

0.022

$<0.001$

5.42

$2.17-9.88$

$<0.001$

0.009

0.15

$0.09-0.25$

0.022

Multivariate

BCLC: Barcelona Clinic Liver Cancer; HR: Hazard ratio; LSM: Liver stiffness measurement; MS: Mean survival.

A multivariate analysis enrolled age and gender of patients, PVTT, tumor size, histological differentiation, Child-Pugh score, BCLC stage, TNM stage and preoperative LSM into the Cox regression model to determine independent prognostic factors for operable HBV-related HCC. The result showed that LSM (HR: 0.15; 95\% CI: 0.09-0.25; $\mathrm{p}<0.05$ ) and TNM (HR: 5.42; 95\% CI: 2.17-9.88; $<<0.05$ ) and pathological differentiation (HR: 0.62; 95\% CI: 0.31-0.95; p < 0.05) and BCLC stage (HR: 7.51; 95\% CI: 2.87-10.54; p < 0.05) and PVTT (HR: 3.80; 95\% CI: 1.23-9.55; p < 0.05) were the independent prognostic factors for OS (Table 2). Furthermore, 


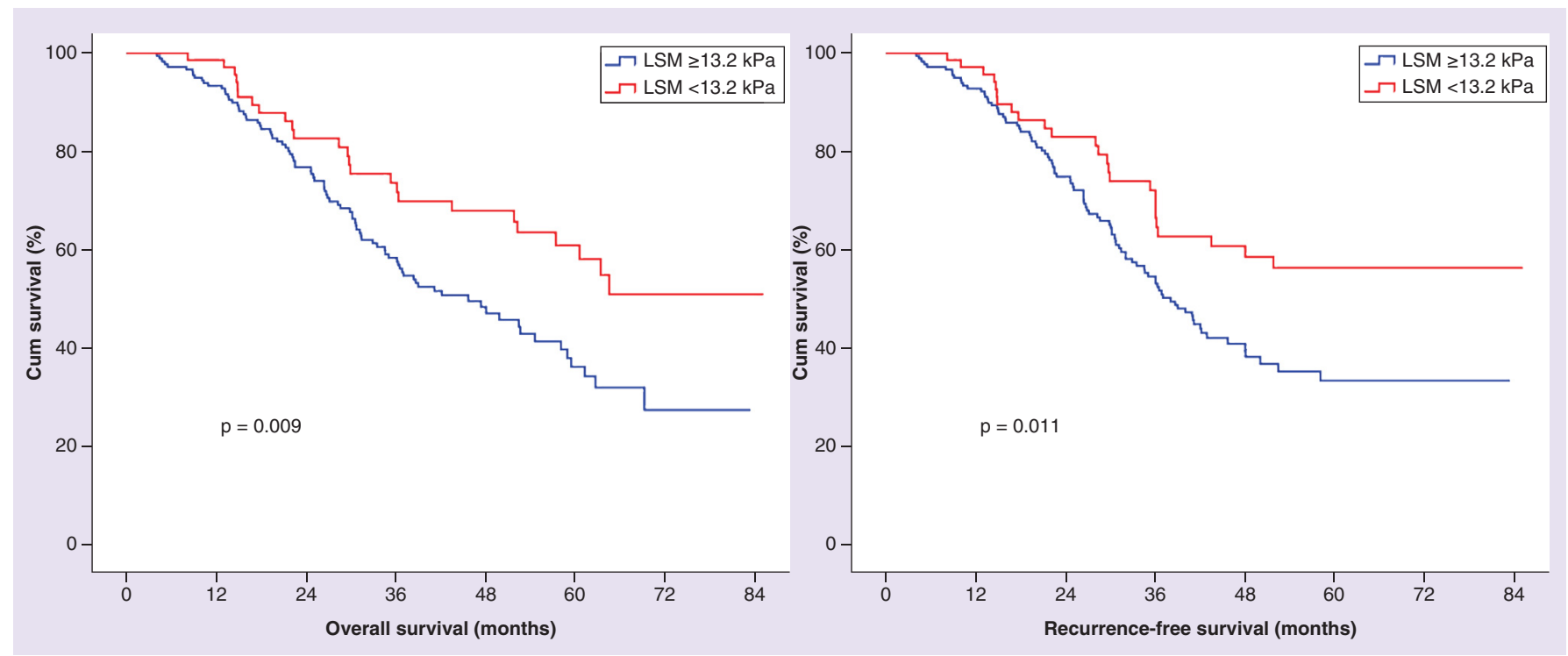

Figure 2. Kaplan-Meier estimates of survival.

LSM (HR: 0.32; 95\% CI: 0.04-1.02; p < 0.05), TNM (HR: 6.51; 95\% CI: 3.71-8.52; $\mathrm{p}<0.05$ ) and BCLC stage (HR: 2.63; 95\% CI: 1.34-6.52; p < 0.05) and PVTT (HR: 5.23; 95\% CI: 2.17-9.32; p < 0.05) were the independent prognostic factors for RFS (Table 3 ).

Relationships of the preoperative LSM with the TNM \& BCLC stages for the prognosis of HCC Furthermore, we also assess the correlation of preoperative LSM with the TNM and BCLC stages for the prognosis of HCC using Kaplan-Meier survival analysis. The OS of patients with an LSM $\geq 13.2 \mathrm{kPa}$ was not significantly poorer than that of patients with an LSM $<13.2 \mathrm{kPa}$ for early stage (TNM stages I and II; 80.3 vs 72.4 months; $\mathrm{p}=0.192$ ), but advanced tumor (TNM stage III; 53.0 vs 44.8 months; $\mathrm{p}=0.016$ ). However, patients with an LSM $<13.2 \mathrm{kPa}$ can obtain better mean RFS comparing to patients with an LSM $\geq 13.2 \mathrm{kPa}$ for early stage (TNM stages I and II; 76.1 vs 60.8 months; $\mathrm{p}=0.041$ ) and advanced tumor (TNM stage III; 56.7 vs 44.0 months; $\mathrm{p}=0.035$; Figure $3 \mathrm{~A}-\mathrm{D})$.

For BCLC stage A HCC, low preoperative LSM $(<13.2 \mathrm{kPa})$ had significant longer overall mean survival than $L S M \geq 13.2 \mathrm{kPa}$ (56.8 vs 45.6 months; $\mathrm{p}=0.042$ ), and this trend remained significant for RFS (55.3 vs 38.8 months; $\mathrm{p}=0.013$ ). Furthermore, patients with an LSM $<13.2 \mathrm{kPa}$ had better mean RFS (62.7 vs 49.1 months; $\mathrm{p}=0.024$ ) and $O S$ (71.0 vs 58.1 months; $\mathrm{p}=0.028$ ) comparing to patients with an LSM $\geq 13.2 \mathrm{kPa}$ for BCLC stages $\mathrm{B}$ and C tumor, see Figure 4.

\section{Discussion}

It has been revealed that there is a significant role of liver fibrosis on progression and metastasis of HCC, especially for patients who had comorbidity of HBV infection [20-22]. In the current study, we found the preoperative LSM value measured though FibroScan was an independent prognostic factor for HBV-related HCC patients. Furthermore, we also found that LSM level was positively correlated with BCLC stage and TNM stage, which means patients with a higher LSM tend to have a more advanced tumor. In subgroup analyses, high LSM is significantly related to poorer RFS for patients with early- and advanced-stage tumors, suggesting that LSM not only can be used to predict survival but also estimate the risk of tumor relapse.

Hepatic fibrosis is considered as a wound-healing response to chronic liver injury, which may result in liver cirrhosis and HCC, thus significantly correlated with prognosis of HCC [23,24]. The extent of hepatic fibrosis has usually been evaluated by histological exanimation. However, there are several limitations in histological evaluation of hepatic fibrosis, including limited accuracy resulted from both sampling variability and interobserver variability between pathologists, and its invasive procedure causing patient discomfort and complications [25,26]. 
Table 3. Univariate and multivariate analyses of the prognostic factors related recurrence-free survival for hepatocellular carcinoma.

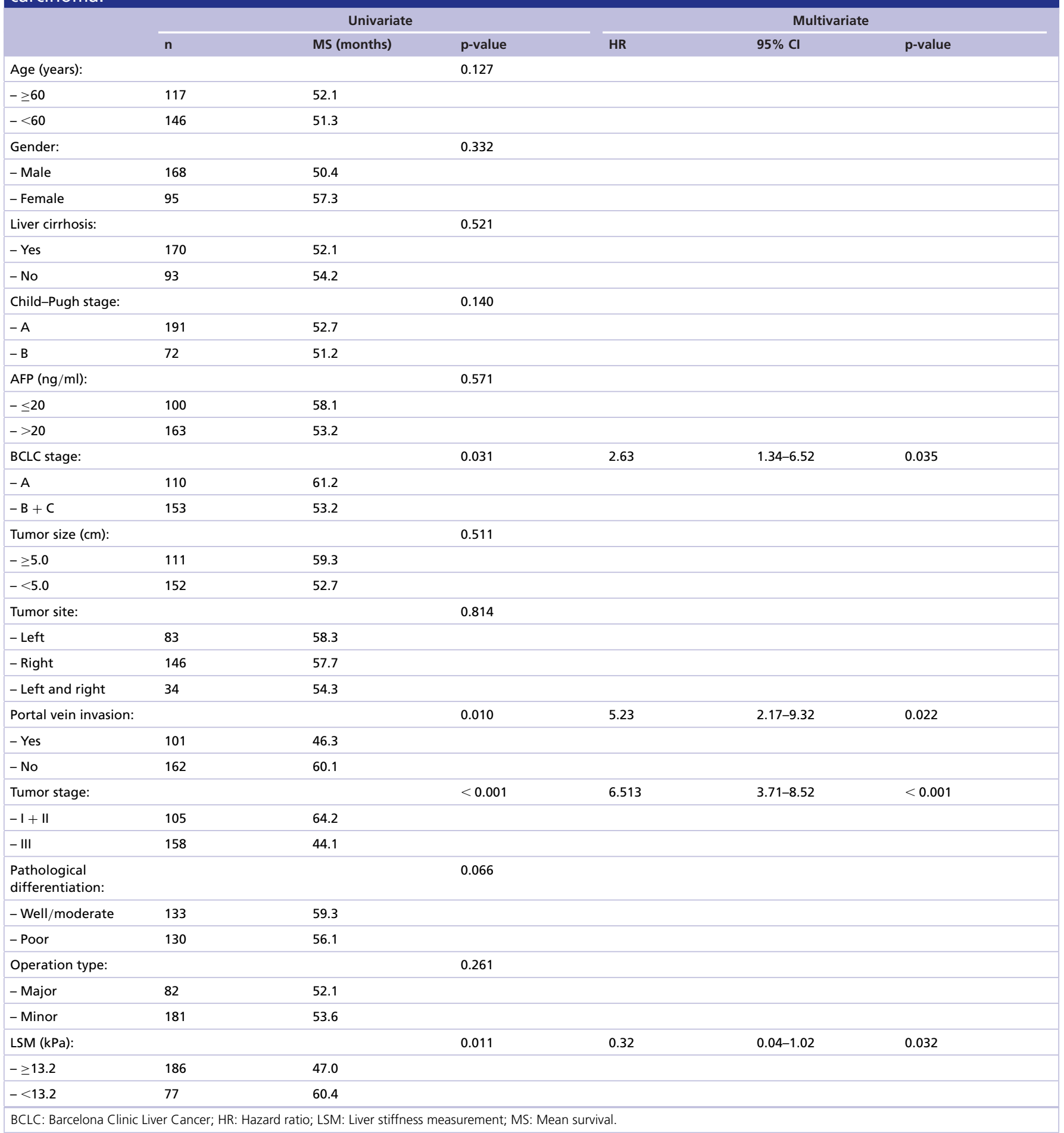

Therefore, several useful non-invasive methods for assessing liver fibrosis have been proposed and confirmed, such as FibroScan [27].

LSM by FibroScan had been recognized as an accurate method for assessing the stage of liver fibrosis in patients with chronic HBV infection [28], indicating values increasing as the liver disease progresses, and relating to HCC patients with cirrhotic background [29]. Jung et al. [17] confirmed the high-preoperative LSM was associated with 


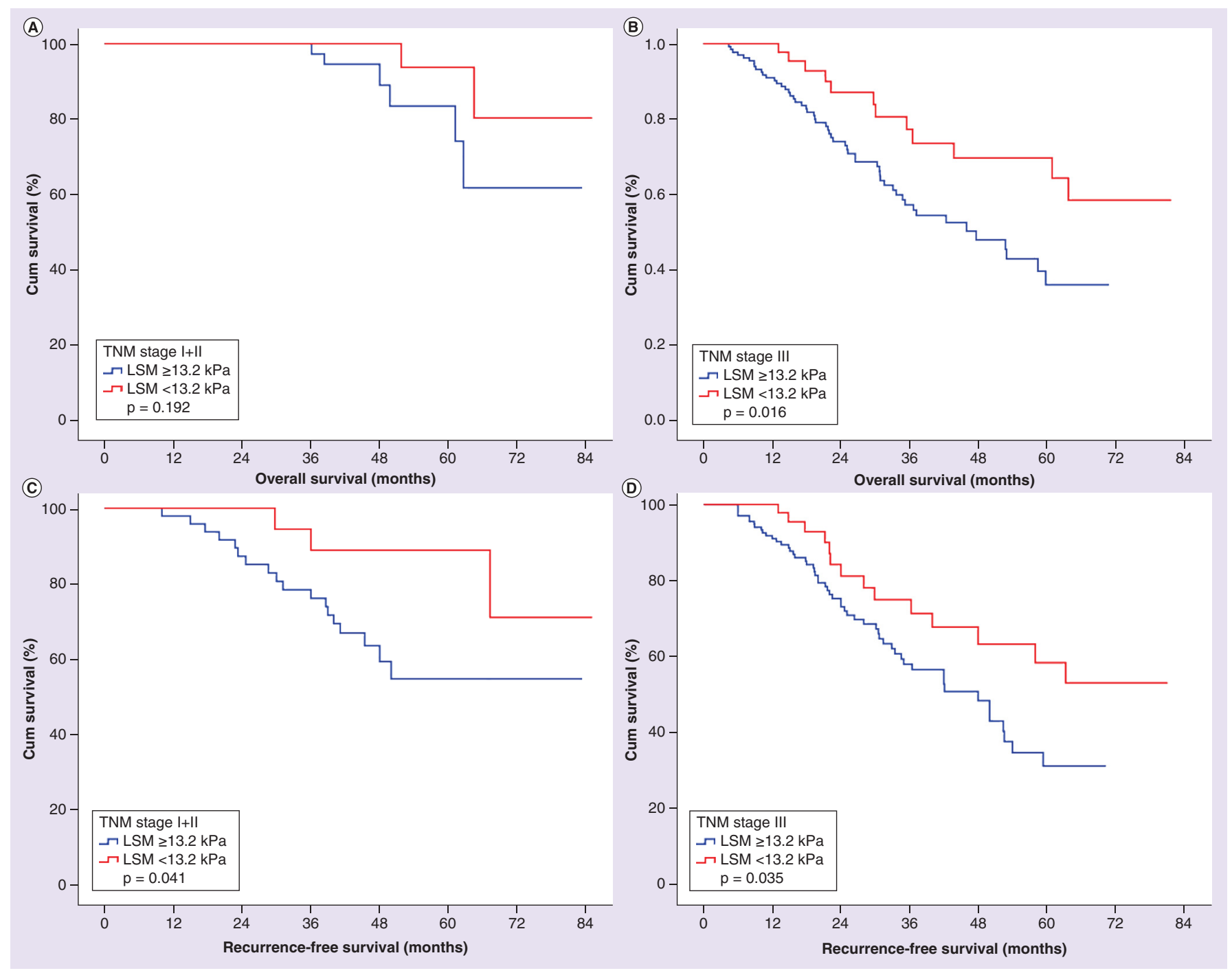

Figure 3. TNM stage-wise survivability.

LSM: Liver stiffness measurement.

significantly greater risk for recurrence of HCC, suggesting LSM can be a useful predictor of recurrence after curative resection of HCC. Therefore, LSM might be a valuable non-invasive tool for assessing the survival prognosis of liver cancer. The most appropriate cut-off value of LSM for diagnosis of liver cirrhosis was 11.8-15.9 kPa with sensitivity ranging $79-87 \%$ and specificity $81-95 \%$ in the HBV and HCV cases [30-32]. However, the cut-off value for liver stiffness most significantly related to HCC remains elusive. A previous study concluded that chronic hepatitis B patients with LSM values more than $12 \mathrm{kPa}$ had a significantly higher risk of HCC development [15]. In addition, HCC patients with pretreatment LSM values more than 13-13.4 kPa experienced a higher incidence of HCC relapse $[17,33]$. Optimal cut-off values were often chosen by using a common optimization step of ROC curve in various evaluation settings. In this study, the best cut-off value of LSM for operable HCC by ROC analysis was $13.2 \mathrm{kPa}$, which was consistent with previous studies. We further confirmed the significance of this cut-off value in stratifying the different spectra of prognosis in patients with HCC development in our study.

It was revealed in this study that LSM value was significantly associated with liver cirrhosis diagnosis. In a clinical setting, liver cirrhosis always has been confirmed based on ultrasonography, laboratory test and radiology imaging, and its accuracy can hardly be evaluated by quantification analysis [34]. Furthermore, the influence of liver cirrhosis may have been confounded, because liver cirrhosis was analyzed as a single category without considering the wide-range severity of liver cirrhosis. However, LSM assesses liver fibrosis accurately and its results, expressed as 


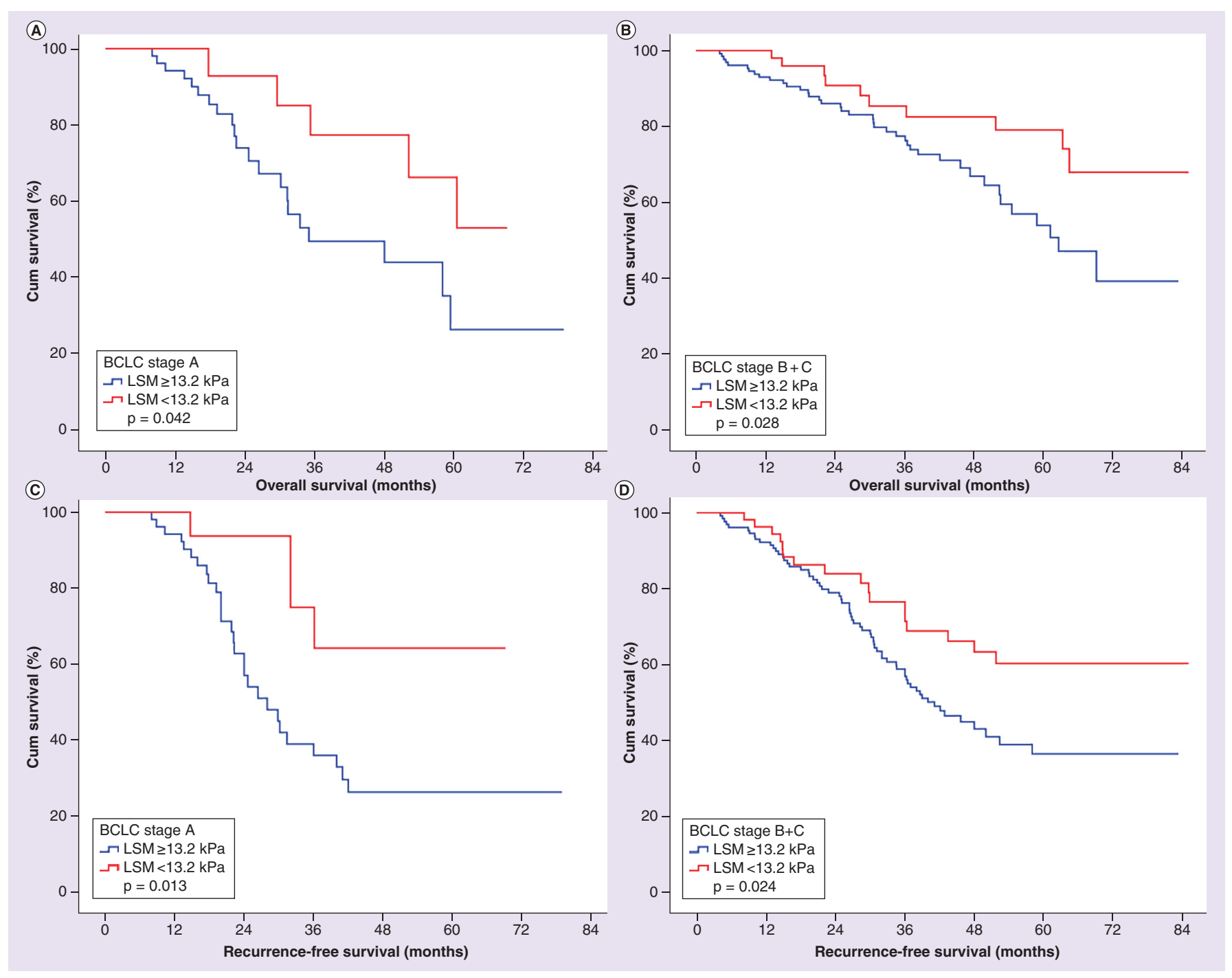

Figure 4. Barcelona Clinic Liver Cancer stage-wise survivability.

BCLC: Barcelona Clinic Liver Cancer; LSM: Liver stiffness measurement.

continuous values, are more helpful for delicate grading of liver fibrosis than simple stepwise histological grading, which also can overcome investigator-dependent limitation [35]. Several studies have demonstrated that hepatitis flares and hypertransaminasemia can lead to high LSM values in spite of low fibrosis, so LSM also reflect the liver function [36,37]. Otherwise, we found that LSM was significantly associated with RFS of patients with early tumor, thereby predicting the relapse risk of early-stage HCC, which is important for patient stratification and individualized treatment. For early-stage patient with high LSM, more aggressive postoperative monitoring and adjunctive therapy should be applied. In such a setting, the relapse can be found as early as possible. The significance of the LSM in the HCC treatment plan needs further randomized controlled trials (RCT).

Our study has some limitations. The retrospective, single-center design of this study may have influenced results, thus contributing to differences between our findings and the previous study. A large multicenter prospective study would be preferred to confirm results and reduce selection bias. Furthermore, the cut-off value of LSM in our study was calculated from a patients group from a single institution, though potentially diminishes generalizability. Moreover, a high level of evidence systemic review and meta-analysis enrolled various validation studies may be required to confirm the cut-off value for LSM for survival of HCC. 


\section{Conclusion}

Our results demonstrated that a low preoperative LSM is a significant predictor of better survival in patients with HCC, which also correlated with BCLC and TNM stages. Future perspective, randomized and control studies with larger samples are required to confirm our results. Currently, LSM by FibroScan, as a quantification accession tool for liver fibrosis, could be considered as an independent prognostic factor for operable and HBV-related HCC.

\section{Future perspective}

Liver fibrosis is closely associated with the development of HCC. The use of LSM may therefore facilitate the liver fibrosis evaluation of patients with HBV-positive hepatocellular carcinoma. The results of our study could potentially guide postoperative management to detect recurrent and improve outcomes.

\section{Summary points}

- The mortality and recurrent rate of patients with hepatocellular carcinoma were 31.5 and $42.6 \%$, respectively.

- In receiver-operating characteristic analysis, optimal cut-off value of liver stiffness measurement (LSM) predicting survival was $13.2 \mathrm{kPa}$, using a sensitivity of $66.3 \%$ and a specificity of $84.7 \%$.

- LSM positively significantly associated with liver cirrhosis, TNM and Barcelona Clinic Liver Cancer stages.

- The mean overall survival (OS) of patients with preoperative LSM $<13.2 \mathrm{kPa}$ was statistically significantly higher than that of those with a LSM $\geq 13.2 \mathrm{kPa}$ (61.3 vs 48.2 months; $p=0.009$ ).

- In univariate analysis of recurrence-free survival (RFS), patients with high LSM also had shorter mean RFS than patients with low LSM (60.4 vs 47.0 months; $p=0.011$ ).

- LSM was confirmed as a significant independent predictor for RFS and OS in multivariate analysis.

- For advanced tumor, LSM was significantly associated to OS and RFS.

- For Barcelona Clinic Liver Cancer stages B and C tumor, LSM was significantly associated to OS and RFS.

Financial \& competing interests disclosure

The authors have no relevant affiliations or financial involvement with any organization or entity with a financial interest in or financial conflict with the subject matter or materials discussed in the manuscript. This includes employment, consultancies, honoraria, stock ownership or options, expert testimony, grants or patents received or pending, or royalties.

No writing assistance was utilized in the production of this manuscript.

Ethical conduct of research

The authors state that they have obtained appropriate institutional review board approval or have followed the principles outlined in the Declaration of Helsinki for all human or animal experimental investigations. In addition, for investigations involving human subjects, informed consent has been obtained from the participants involved.

Open access

This work is licensed under the Attribution-NonCommercial-NoDerivatives 4.0 Unported License. To view a copy of this license, visit http://creativecommons.org/licenses/by-nc-nd/4.0/

\section{References}

1 Siegel RL, Miller KD, Jemal A. Cancer statistics, 2016. CA Cancer J. Clin. 66(1), 7-30 (2016).

2 Chen W, Zheng R, Baade PD et al. Cancer statistics in China, 2015. CA Cancer J. Clin. 66(2), 115-132 (2016).

3 Liu C, Chang L, Ji H et al. Prevalence of HBV DNA among 20 million seronegative blood donations in China from 2010 to 2015. Sci. Rep. 6, 36464 (2016).

4 Poon RT. Prevention of recurrence after resection of hepatocellular carcinoma: a daunting challenge. Hepatology 54(3), 757-759 (2011).

5 Tralhao JG, Dagher I, Lino T, Roudie J, Franco D. Treatment of tumour recurrence after resection of hepatocellular carcinoma. Analysis of 97 consecutive patients. Eur. J. Surg. Oncol. 33(6), 746-751 (2007).

6 Della Corte C, Aghemo A, Colombo M. Individualized hepatocellular carcinoma risk: the challenges for designing successful chemoprevention strategies. World J. Gastroenterol. 19(9), 1359-1371 (2013).

7 Zampino R, Pisaturo MA, Cirillo G et al. Hepatocellular carcinoma in chronic HBV-HCV co-infection is correlated to fibrosis and disease duration. Ann. Hepatol. 14(1), 75-82 (2015).

8 Li Z, Zhao X, Jiang P et al. HBV is a risk factor for poor patient prognosis after curative resection of hepatocellular carcinoma: a retrospective case-control study. Medicine (Baltimore) 95(31), e4224 (2016). 
9 Chin JL, Pavlides M, Moolla A, Ryan JD. Non-invasive markers of liver fibrosis: adjuncts or alternatives to liver biopsy? Front. Pharmacol. 7, 159 (2016).

10 Amarapurkar D. Role of liver biopsy in management of chronic hepatitis B and chronic hepatitis C. Trop. Gastroenterol. 28(2), 67-68 (2007).

11 Bartres C, Lens S. Elastography of the liver (Fibroscan[R]) in hepatology. Rev. Esp. Enferm. Dig. 105(4), 235 (2013).

12 Fernandez M, Trepo E, Degre D et al. Transient elastography using Fibroscan is the most reliable noninvasive method for the diagnosis of advanced fibrosis and cirrhosis in alcoholic liver disease. Eur. J. Gastroenterol. Hepatol. 27(9), 1074-1079 (2015).

13 Foucher J, Chanteloup E, Vergniol J et al. Diagnosis of cirrhosis by transient elastography (FibroScan): a prospective study. Gut 55(3), 403-408 (2006).

14 Feier D, Lupsor Platon M, Stefanescu H, Badea R. Transient elastography for the detection of hepatocellular carcinoma in viral C liver cirrhosis. Is there something else than increased liver stiffness? J. Gastrointestin. Liver Dis. 22(3), 283-289 (2013).

15 Tatsumi A, Maekawa S, Sato M et al. Liver stiffness measurement for risk assessment of hepatocellular carcinoma. Hepatol. Res. 45(5), 523-532 (2015).

16 Berzigotti A, Reig M, Abraldes JG, Bruix J, Bosch J, Garcia-Pagan JC. Value of transient elastography measured with fibroscan in predicting the outcome of hepatic resection for hepatocellular carcinoma. Ann. Surg. 261(4), e105 (2015).

17 Jung KS, Kim SU, Choi GH et al. Prediction of recurrence after curative resection of hepatocellular carcinoma using liver stiffness measurement (FibroScan[R]). Ann. Surg. Oncol. 19(13), 4278-4286 (2012).

18 Strasberg SM. Nomenclature of hepatic anatomy and resections: a review of the Brisbane 2000 system. J. Hepatobiliary Pancreat. Surg. 12(5), 351-355 (2005).

19 Li GJ, Ji JJ, Yang F, Xu HW, Bai Y. Preoperative lymphocyte-to-monocyte ratio predicts survival in primary hepatitis B virus-positive hepatocellular carcinoma after curative resection. Onco. Targets Ther. 10, 1181-1189 (2017).

20 Xiao G, Zhu F, Wang M et al. Diagnostic accuracy of APRI and FIB-4 for predicting hepatitis B virus-related liver fibrosis accompanied with hepatocellular carcinoma. Dig. Liver Dis. 48(10), 1220-1226 (2016).

21 Wang Q, Blank S, Fiel MI et al. The severity of liver fibrosis influences the prognostic value of inflammation-based scores in hepatitis B-associated hepatocellular carcinoma. Ann. Surg. Oncol. 22(Suppl. 3), S1125-S1132 (2015).

22 Suh B, Park S, Shin DW et al. High liver fibrosis index FIB-4 is highly predictive of hepatocellular carcinoma in chronic hepatitis B carriers. Hepatology 61(4), 1261-1268 (2015).

23 Wang Q, Fiel MI, Blank S et al. Impact of liver fibrosis on prognosis following liver resection for hepatitis B-associated hepatocellular carcinoma. Br. J. Cancer 109(3), 573-581 (2013).

24 Fuchs BC, Hoshida Y, Fujii T et al. Epidermal growth factor receptor inhibition attenuates liver fibrosis and development of hepatocellular carcinoma. Hepatology 59(4), 1577-1590 (2014).

25 Regev A, Berho M, Jeffers LJ et al. Sampling error and intraobserver variation in liver biopsy in patients with chronic HCV infection. Am. J. Gastroenterol. 97(10), 2614-2618 (2002).

26 Bedossa P, Dargere D, Paradis V. Sampling variability of liver fibrosis in chronic hepatitis C. Hepatology 38(6), 1449-1457 (2003).

27 Xie QX, Xu N, Jiang XP et al. Role of FibroScan in liver fibrosis evaluation in patients with chronic hepatitis B virus infection and related influencing factors. Zhonghua Gan Zang Bing Za Zhi 24(9), 659-664 (2016).

28 Kettaneh A, Marcellin P, Douvin C et al. Features associated with success rate and performance of FibroScan measurements for the diagnosis of cirrhosis in HCV patients: a prospective study of 935 patients. J. Hepatol. 46(4), 628-634 (2007).

29 Adler M, Larocca L, Trovato FM, Marcinkowski H, Pasha Y, Taylor-Robinson SD. Evaluating the risk of hepatocellular carcinoma in patients with prominently elevated liver stiffness measurements by FibroScan: a multicentre study. HPB (Oxford) 18(8), 678-683 (2016).

30 Akima T, Tamano M, Hiraishi H. Liver stiffness measured by transient elastography is a predictor of hepatocellular carcinoma development in viral hepatitis. Hepatol. Res. 41(10), 965-970 (2011).

31 Sultanik P, Sogni P, Meritet JF, Pol S, Mallet V. Patients with chronic hepatitis B should be screened for hepatocellular carcinoma regardless of liver stiffness measurement. Hepatology 63(2), 672 (2016).

32 Liu XY, Ma LN, Yan TT et al. Combined detection of liver stiffness and C-reactive protein in patients with hepatitis B virus-related liver cirrhosis, with and without hepatocellular carcinoma. Mol. Clin. Oncol. 4(4), 587-590 (2016).

33 Lee SH, Kim SU, Jang JW et al. Use of transient elastography to predict de novo recurrence after radiofrequency ablation for hepatocellular carcinoma. Onco. Targets Ther. 8, 347-356 (2015).

34 Ge L, Shi B, Song YE, Li Y, Wang S, Wang X. Clinical value of real-time elastography quantitative parameters in evaluating the stage of liver fibrosis and cirrhosis. Exp. Ther. Med. 10(3), 983-990 (2015).

35 Wang JH, Chuah SK, Lu SN et al. Baseline and serial liver stiffness measurement in prediction of portal hypertension progression for patients with compensated cirrhosis. Liver Int. 34(9), 1340-1348 (2014). 
36 Coco B, Oliveri F, Maina AM et al. Transient elastography: a new surrogate marker of liver fibrosis influenced by major changes of transaminases. J. Viral Hepat. 14(5), 360-369 (2007).

37 Sagir A, Erhardt A, Schmitt M, Haussinger D. Transient elastography is unreliable for detection of cirrhosis in patients with acute liver damage. Hepatology 47(2), 592-595 (2008). 